\title{
COMPACTNESS OF LIMIT SETS AND SEMIORBIT CLOSURES
}

ETHAN M. COVEN

1. Introduction. The notion of the $P$-limit set $P_{x}$ of a point is a natural generalization of that of the omega limiting set of a point. (See [1] and [2]. See [2] for notation and terminology.) It is well known that $P_{x}$ is compact and nonempty if the corresponding semiorbit $x P$ has compact closure. In this paper we establish (Theorem 2) the converse of this statement for transformation groups with locally compact Hausdorff phase space and generative phase group. We introduce the concept of $K$-connectedness and show (Theorem 1 ) that a replete semigroup has a translate which is $K$-connected. This is a generalization of a result of F. J. Hahn [3, Lemma 3.6] on replete semigroups in $R^{n}$.

2. $K$-connectedness. Let $T$ be a generative topological group. Thus $T$ is abelian and is generated by a compact neighborhood $K$ of the identity, i.e., $T=\bigcup_{n=0}^{\infty} K^{n}$. We may assume without loss of generality that $K$ is symmetric. We now fix $K$ for the remainder of this section.

Let $P$ be a replete semigroup in $T$. Define an equivalence relation $\sim$ on $P$ as follows: $a \sim b$ provided that there exist $k_{1}, \cdots, k_{n} \in K$ such that

(1) $b=a k_{1} \cdots k_{n}$.

(2) $a k_{1} \cdots k_{i} \in P$ for $i=1,2, \cdots, n$.

Definition. A subset $Q$ of $P$ is said to be $K$-connected provided that all the elements of $Q$ belong to the same equivalence class.

REMARK. (1) Any subset of a $K$-connected set is $K$-connected.

(2) If $A$ and $B$ are $K$-connected and if $A \cap B \neq \varnothing$, then $A \cup B$ is $K$-connected.

(3) If $A_{1}, A_{2}, \cdots$ are $K$-connected and if $A_{n} \cap A_{n+1} \neq \varnothing$ for $i=1,2, \cdots$, then $\bigcup_{n=1}^{\infty} A_{n}$ is $K$-connected.

(4) If $p \in P$ and $A$ is $K$-connected, then $p A$ is $K$-connected.

(5) If $p \in P$ and $p K^{n} \subseteq P$, then $p K^{n}$ is $K$-connected.

Lemma 1. Let $Q$ be a $K$-connected replete semigroup contained in $P$. Then $P Q$ is K-connected.

Received by the editors February 27, 1968. 
Proof. Let $p_{1}, p_{2} \in P$. Since $Q$ is a replete semigroup, $T=Q Q^{-1}$ [2, Theorem 6.04]. Hence $p_{1} p_{2}^{-1} \in Q Q^{-1}$ and so $p_{1} Q \cap p_{2} Q \neq \varnothing$. Now $p_{1} Q$ and $p_{2} Q$ are both $K$-connected, so $p_{1} Q \cup p_{2} Q$ is $K$-connected. But $p_{1}$ and $p_{2}$ were chosen arbitrarily, so $\bigcup_{p \in P} p Q=P Q$ is $K$-connected.

Theorem 1. There exists $p \in P$ such that $p P$ is $K$-connected.

Proof. It suffices to find a $K$-connected replete semigroup $Q$ contained in $P$. For then $p P \subseteq P Q$ for any $p \in Q$.

Choose $p \in P$ such that $p K \subseteq P$. Then $p \in K^{m}$ for some $m \geqq 0$. Let $Q=\bigcup_{n=m}^{\infty} p^{n} K^{n}$. Then $Q$ is a replete semigroup [2, Theorem 6.08] and it is clearly contained in $P$. We show that $Q$ is $K$-connected.

Since $p \in K^{m}$ and $e \in K^{m+1}$, it follows that $p^{m} K^{m} \cap p^{m+1} K^{m+1} \neq \varnothing$, as $p^{m+1}$ is in both sets. Now let $j \geqq 0$. Then $p^{m+j} K^{m} \cap p^{m+j+1} K^{m+1} \neq \varnothing$, and so $p^{m+j} K^{m+j} \cap p^{m+j+1} K^{m+j+1} \neq \varnothing$. Hence by (3) and (5) of the Remark, $Q$ is $K$-connected.

Definition. (See [1].) A subset $E$ of $P$ is said to be $P$-extensive provided that $E \cap p P \neq \varnothing$ for all $p \in P$.

Corollary. Let $P_{1}$ and $P_{2}$ be subsets of $P$ such that no member of $P_{1}$ is in the same equivalence class as any member of $P_{2}$. Then $P_{1}$ and $P_{2}$ cannot both be P-extensive.

Lemma 2. Let $P_{1}$ and $P_{2}$ be disjoint subsets of $P$ such that $P_{1} \cup P_{2}=P$. Then the following statements are equivalent:

(1) $P_{1} K \cap P_{2}=\varnothing$.

(2) No member of $P_{1}$ is in the same equivalence class as any member of $P_{2}$.

Proof. (2) $\Rightarrow(1)$. Let $p_{1} \in P_{1}, k \in K$. If $p_{1} k \in P$, then $p_{1} k \sim p_{1}$, hence $p_{1} k \notin P_{2}$. If $p_{1} k \notin P$, then $p_{1} k \notin P_{2}$.

(1) $\Rightarrow(2)$. Let $p_{1} \in P_{1}, p_{2} \in P_{2}$ and $p_{1} \sim p_{2}$. Thus there exists $k_{1}, \cdots$, $k_{n} \in K$ such that $p_{1} k_{1} \cdots k_{n}=p_{2}$ and $p_{1} k_{1} \cdots k_{i} \in P$ for $i=1$, $2, \cdots, n$. Hence $p_{1} k_{1} \in P_{1} K \cap P=P_{1}$, and so $p_{1} k_{1} k_{2}=\left(p_{1} k_{1}\right) k_{2} \in P_{1}$. Similarly $p_{1} k_{1} \cdots k_{i} \in P_{1}$ for $i=1,2, \cdots, n$. But this contradicts $p_{2} \in P_{2}$.

3. Theorem on compactness of limit sets and semiorbit closures.

Definition. (See [2].) Let $x \in X$ and let $P$ be a replete semigroup in $T$. Then the $P$-limit set of $x$ is defined by

$$
P_{x}=\bigcap_{t \in T} \operatorname{cl}(x t P) .
$$

It is easy to show that $P_{x}$ is closed and invariant, and that $P_{x}=\bigcap_{p \in P} \operatorname{cl}(x p P)$. 
THEOREM 2. Let $(X, T, \pi)$ be a transformation group where $X$ is a locally compact Hausdorff space and $T$ is generative. Let $x \in X$ and let $P$ be a replete semigroup in $T$. Then $P_{x}$ is compact and nonempty if and only if $\operatorname{cl}(x P)$ is compact.

Proof. It suffices to show that if $P_{x}$ is compact and nonempty, then $\operatorname{cl}(x P)$ is compact. So, let $U$ be an open neighborhood of $P_{x}$ which has compact closure, and let $K$ be a compact symmetric neighborhood of the identity which generates $T$. Let $A=\bar{U} K-U$, and so $A$ is compact. For each $p \in P$, let $A(p)=A \cap \operatorname{cl}(x p P)$. Thus each $A(p)$ is a compact subset of $A$. Now

$$
\bigcap_{p \in P} A(p)=A \cap \bigcap_{p \in P} \operatorname{cl}(x p P)=A \cap P_{x}=\varnothing
$$

so by the finite intersection property, there exist $p_{1}, p_{2}, \cdots, p_{n} \in P$ such that $A\left(p_{1}\right) \cap \cdots \cap A\left(p_{n}\right)=\varnothing$. Let $p_{0}=p_{1} p_{2} \cdots p_{n}$ and thus $A\left(p_{0}\right)=\varnothing$. Hence $x p_{0} P \subseteq X-A=U \cup(X-\bar{U} K)$. Let

$$
P_{1}=\left\{p \in P \mid x p_{0} p \in U\right\} \quad P_{2}=\left\{p \in P \mid x p_{0} p \in X-\bar{U} K\right\} .
$$

Then $P_{1} \cup P_{2}=P$ and $P_{1} \cap P_{2}=\varnothing$.

Let $p_{1} \in P_{1}$ and $k \in K$. Then $x p_{0} p_{1} k \in U K$ and so $p_{1} k \notin P_{2}$. Thus $P_{1} K \cap P_{2}=\varnothing$ and then by Lemma 2 , no member of $P_{1}$ is in the same equivalence class as any member of $P_{2}$. Hence by the corollary, $P_{1}$ and $P_{2}$ cannot both be $P$-extensive.

We now show that $P_{1}$ is $P$-extensive. Since $P_{x}$ is invariant, $U p_{0}^{-1}$ is a neighborhood of $P_{x}$. As $P_{x} \neq \varnothing,\left\{p \in P \mid x p \in U p_{0}^{-1}\right\}$ is $P$-extensive. However this last set is precisely $P_{1}$. Thus we conclude that $P_{2}$ is not $P$-extensive, i.e., that there exists $p \in P$ such that $P_{2} \cap p P=\varnothing$. Hence $p P \subseteq P_{1}$. Then $x p P \subseteq x P_{1} \subseteq U p_{0}^{-1}$. Thus $x P \subseteq U p_{0}^{-1} p^{-1}$ and so $\mathrm{cl}(x P)$ is compact.

\section{REFERENCES}

1. John D. Baum, P-recurrence in topological dynamics, Proc. Amer. Math. Soc. 7 (1956), 1146-1154.

2. W. H. Gottschalk and G. A. Hedlund, Topological dynamics, Amer. Math. Soc. Colloq. Publ. no. 36, Amer. Math. Soc., Providence, R. I., 1956.

3. Frank J. Hahn, Some embeddings, recurrence properties, and the Birkhoff-Markov Theorem, Duke Math. J. 27 (1960), 513-525.

WESLEYAN UNIVERSITY 